\title{
Prevalence of hypertension and its risk factor among cotton textile workers in low-and-middle- income countries: A systematic review protocol
}

Naureen Ali ( $\sim$ naureenalimeghani@gmail.com )

Aga Khan University Hospital

Anam Feroz

Aga Khan University Medical College Pakistan

\section{Protocol}

Keywords: Blood pressure, Hypertension, Risk-factors, Prevalence, Adult workers, Low and Middle Income Countries, Systematic Review, Cotton textile workers

Posted Date: April 15th, 2020

DOI: https://doi.org/10.21203/rs.2.24338/v3

License: (c) (1) This work is licensed under a Creative Commons Attribution 4.0 International License.

Read Full License

Version of Record: A version of this preprint was published at Systematic Reviews on May 2nd, 2020. See the published version at https://doi.org/10.1186/s13643-020-01364-z. 


\section{Abstract}

Background: Cotton workers are exposed to various hazards in the textile industry that might result in different ailments including hypertension (HTN). However, few attempts have been made to systematically review the prevalence of hypertension and its risk factor among cotton textile workers in low-and-middle-income countries (LMICs). The objective of this study will be to evaluate the prevalence of hypertension and its risk factors among adult cotton textile workers in low- and middle-income countries.

Methods: We designed and registered a study protocol for a systematic review of descriptive epidemiology data. We will include observational studies (e.g. cross-sectional, cohort, surveys) on the epidemiology of hypertension among adult cotton textile workers in low- and middle-income countries. The primary outcome will be the prevalence of hypertension. Secondary outcomes will be the prevalence of risk factors of hypertension. Literature searches will be conducted in multiple electronic databases (from January 2000 onwards), including PubMed/MEDLINE, CINAHL, Science Direct, and Cochrane Library. Grey literature will be identified through searching conference abstracts, thesis dissertations and public repositories. Two investigators will independently screen all citations, full-text articles, and abstract data. The study methodological quality (or bias) will be appraised using an appropriate tool. If feasible, we will conduct random effects meta-analysis. Additional analyses will be conducted to explore the potential sources of heterogeneity (e.g. age, gender, years of service, textile department).

Discussion: This review will highlight the proportion of hypertension along with its risk factor among cotton textile workers in LMICs. Given that, some of the factors can be prevented in this high-risk population, we will call on health, experts to prioritize policies and commission and conduct programs to support the improvement in their health. This systematic review will identify, evaluate and integrate evidence on the prevalence and risk factors of hypertension among adult cotton textile workers in lowand middle-income countries. Our findings will be made publicly available in a repository and published in a peer-reviewed journal

Systematic Review Registration: The present protocol has been registered within the PROSPERO database (registration ID: CRD42020167175)

\section{Background}

Globally, hypertension (HTN) is identified as one of the leading causes of mortality and disability [1]. It has led to 7.5 million deaths and accounted for 57 million disability adjusted life years (DALYS) or 3.7\% of total DALYS[2]. Hypertension is characterized as constant rise of systolic blood pressure and/or diastolic blood pressure of more than or equivalent to $140 \mathrm{mmHg}$ and/or $90 \mathrm{mmHg}$ respectively[3]. Currently, around 1.13 billion individuals are suffering from hypertension globally and two-third of them resides in low- and middle-income countries (LMICs) [4] while, LMICs selection is based on World Bank's (WB) 2018 Country Classification list, having GNI per capita between $\$ 996$ and $\$ 3,895$ [5]. The number of 
hypertensive people is projected to grow by 70 million individual from 2000 to 2025 in high income countries (HIC) while it is estimated to grow by $>500$ million in LMICs over the same period [6]. A systematic review included data on 1,494,609 adults from 45 countries also showed that there is the highest burden of hypertension (32\%) in LMICs and prevalence estimates were found to be higher in elders, overweight/obese, urban settlers and having informal education [7]. Moreover, worldwide high blood pressure is one of the major risk factors for cardiovascular diseases (CVD) that refers to diseases of the heart and blood vessels [8, 9],stroke, coronary artery disease and peripheral vascular disease [9]. The burden of CVD across the world is very high and in LMICs, $47 \%$ of mortality secondary to CVDs and $44 \%$ of CVDs burden are attributable to HTN/high blood pressure (BP) $[9,10]$. Generally, it is asymptomatic and the primary presentation can be seen with a devastating event like stroke or heart disease [11]. It is estimated that $45 \%$ of deaths with heart disease, and $51 \%$ of deaths with stroke are related to hypertension [12]. This is the reason that it is referred to as the "silent killer" [11].

Worldwide, the textile industry has more than 60 million employees[13] who are involved in the production of fibers, yarns, fabrics, clothing and textile products for domestic and decoration, along with the technical and industrial purposes [14]. Moreover, the textile industry involves a cluster of related industries that utilizes a range of natural fibers (cotton, wool, silk, etc.) and/or synthetic fibers to generate fabric[15] which eventually produces varying amounts of dust [16]. Workers are exposed to various hazards in textile industry that might result in different ailments[17, 18]. Studies reveal high level of oxidative stress in cotton textile workers [19]leading to the development of essential hypertension [20, 21]. A study conducted in Kannur district in India found out that the prevalence of hypertension was $22.3 \%$ among cotton textile workers which was equivalent to the prevalence of general population [22]. Furthermore, increasing age, alcohol consumption, family history of hypertension, BMI $>25 \mathrm{~kg} / \mathrm{m} 2$ and high waist-hip ratio were identified as a strong factor of hypertension among cotton workers[22]. Similarly, a study conducted in Dhaka, Bangladesh notified that both general and central obesity were found to be significantly associated with hypertension [23]. Additionally, factors such as work-related stress, noise pollution, lack of earplug usage, tobacco smoking, and long duration of employment contributes towards the development of hypertension among the cotton textile workers. [22, 24-27]. While, stress in job also have a negative impact on worker's cardiovascular health which in turn will affect their job performance [28]. However, the association between cotton dust and increased blood pressure may not be strong, as confounding factors and bias impact could not be excluded $[18,29]$

Chronic diseases make workers less productive, more prone to injury that leads to significant suffering and, add huge financial and service challenges on health care systems [23, 30-32]. Furthermore, it is witnessed that collective monetary losses related with the hypertension, \& diabetes mellitus (DM), in low and middle-income countries are estimated to be over \$7 trillion during the years 2011-2025 that move millions of individuals below the poverty line [33]. Hypertension accounts for the global burden of cardiovascular disease which evidently have huge impact on individuals, organizations and country in terms of economy [11]. Therefore, early detection of hypertension by identifying its risk factors is essential among high risk population particularly in LMIC. Thus, the objective of this study will be to 
evaluate the prevalence of hypertension and its risk factors among adult cotton textile workers in lowand middle-income countries.

\section{Methods}

The present protocol has been registered within the PROSPERO database (registration ID: CRD42020167175). The present study protocol is being reported in accordance with the reporting guidance provided in the Preferred Reporting Items for Systematic Reviews and Meta-Analyses Protocols (PRISMA-P) statement [34]. (See PRISMA-P checklist in Additional file 1).

\section{Eligibility criteria:}

Studies will be selected according to the following criteria: participants, outcome(s) of interest, study design and context.

- Participants (population): We will include studies involving adult cotton textile workers (regardless of gender). We will exclude adults working in another industries (e.g. oil mill, steel mill and coal mill).

- Condition or outcome(s) of interest. The primary outcome will be the prevalence of hypertension (or high blood pressure) indicating the number of people that have the condition divided by the population number at a given point in time. This is often presented as a (prevalence) proportion. We will use authorreported definitions (according to definitions used in the included studies e.g. accepted diagnostic criteria or self-report). Secondary outcomes will be the individual risk factor of hypertension. Potential individual risk factors may include: being overweight or obese, physically inactive, using tobacco, excessive alcohol intake, excessive salt (sodium) in the diet, too little potassium in the diet, stress, or certain chronic conditions (such as kidney disease, diabetes and sleep apnea).

- Study design and context. Eligible studies will be observational studies (cohort, cross-sectional or health surveys) reporting prevalence data using validated or non-validated tools and conducted in low- and middle-income countries. Cross-sectional studies will be the most appropriate study design to determine the prevalence of hypertension. Cross-sectional health surveys are typically used to estimate the point prevalence of common conditions of long duration. For cohort studies, only the first phase (crosssectional) data will be considered. We will exclude randomized trials, quasi-experimental studies, case studies, case series, qualitative studies, systematic review, protocols, commentaries, and editorials.

- Context/settings. We will include studies conducted in low- and middle-income countries.

No limitations will be imposed on publication status (unpublished studies will be eligible for inclusion e.g. conference proceedings, abstracts). We will only include studies published in English, from January 2000 onwards.

\section{Information sources and search strategy}

The primary source of literature will be a structured search of major electronic databases (from January 2000 onwards): MEDLINE (PubMed), CINAHL Plus, Science Direct, and Cochrane Library. The secondary source of potentially relevant material will be a search of the grey or difficult to locate literature, including conference abstracts from selected national or international meeting, thesis dissertations or documents 
in public repositories. We will perform hand-searching of the reference lists of included studies, relevant reviews, or other relevant documents. The literature searches will be designed and conducted by the review team. The search terms will be grouped into following categories of interest: population (e.g. cotton textile, workers, factory workers, and cotton workers), epidemiological studies (e.g. prevalence, cross-sectional), outcomes (e.g. hypertension, blood pressure, systolic blood pressure, diastolic blood pressure, hypertension, high blood pressure and HTN, risk factors, factors, predictors), and settings (e.g. low- and middle-income countries). Additionally, indexed keywords in the Medical Subject Headings $(\mathrm{MeSH})$ will be used in order to ensure uniform search terms. The search strategy will be piloted to ensure sufficient specificity and sensitivity. A draft search strategy for PubMed/MEDLINE is provided in Additional file 2.

\section{Screening and selection of studies:}

All articles identified from the literature search will be uploaded into EndNote [35]. Records will be screened by two reviewers (NA \& AF) independently. A pre-defined screening tool will be designed, and a pilot testing will be conducted. First, titles and abstracts of articles returned from initial searches will be screened based on the eligibility criteria outlined above. Second, full texts will be examined in detail and screened for eligibility. Third, references of all considered articles will be hand-searched to identify any relevant report missed in the search strategy. Any disagreements will be resolved by discussion to meet a consensus, if necessary. We will contact the primary author of the study through email in case of any incomplete or missing information. A flow chart showing details of studies included and excluded at each stage of the study selection process will be provided

\section{Data collection process}

Data will be extracted onto a customized sheet in Excel that will be pilot tested prior to initiating the data extraction process. This sheet will be completed by two independent reviewers (NA, AF) for the eligible studies. Data extraction tables filled by two reviewers will be compared to confirm that all main findings are included. During the data extraction process a third assessor (SF) will be involved if any discordant data is witnessed. A pilot data extraction table is provided in Additional file 3. Besides, existing studies on this research domain have been revised to assess items in the extraction form. The form included the primary author, year of publication, study setting, country of study, objective, study design, study population, male proportion, average age, BP measurement method, BP measurement apparatus, prevalence of hypertension, average BP risk-factors of hypertension co-morbidities, study limitations, included/excluded, and reason for exclusion, quality appraisal of included studies and reviewer name,.

\section{Evaluation of study quality}

The quality of the included studies will be evaluated by standardized quality assessment tool which will be conducted by two authors (NA, AF). Mixed Methods Appraisal Tool (MMAT) tool will be used to evaluate the methodological quality of all non-randomized studies[36]. This tool is used to assess several aspects like selection of study participant, study tool, exposure period, missing data, measurement in 
outcomes, and selection of the reported result. Two reviewer (NA, AF) will rate each study as critical, serious, moderate, or low risk of bias via judgment of the gathered information. If there is limited information then the risk of bias will be categorized as "no information" or the reviewer will contact corresponding study authors for complete information.

\section{Synthesis of included studies}

The data from each paper (e.g. study characteristics, context, participants, outcomes and findings) will be used to build evidence tables of an overall description of included studies. Crude prevalence estimates (number of cases/sample size) will be presented along with $95 \%$ confidence intervals. If feasible and appropriate, prevalence data from primary observational studies will be used to perform random effects meta-analyses. Since heterogeneity is expected a priori, we will estimate the pooled prevalence using the random effects model. The random effects model assumes the study prevalence estimates follow a normal distribution, considering both within-study and between-study variation. Forest plots will be used to visualize the extent of heterogeneity among studies. We will quantify statistical heterogeneity by estimating the variance between studies using $\mathrm{I}^{2}$ statistic. The $\mathrm{I}^{2}$ is the proportion of variation in prevalence estimates that is due to genuine variation in prevalence rather than sampling (random) error. $1^{2}$ ranges between $0 \%$ and $100 \%$ (with values of $0-25 \%$ and $75-100 \%$ taken to indicate low and considerable heterogeneity, respectively). We will also report Cochran $Q$ test with a $P$ value of $<0.05$ considered statistically significant. If sufficient studies are identified and data points are available, potential sources of heterogeneity will be investigated further by subgroup analyses according to baseline characteristics and methodological covariates. We plan to conduct analyses by gender (male vs. female), age (e.g. young adult, middle-age adult, vs. older adult), years of service, and textile department.

\section{Meta-biases:}

If data permits, small study effects (publication bias) will be assessed by inspection of the funnel plots for asymmetry and with Egger's test and Begg's test, with the results considered to indicate potential small study effects when $P$ values $<0.10$.

\section{Discussion}

This review will highlight the proportion of hypertension along with its risk factor among cotton textile workers in LMICs. Given that, some of the modifiable factors may be prevented in this high risk population, we will call on health experts including policy makers, stake holders, cardiovascular epidemiologist and occupational health and safety specialists to prioritize policies and commission and conduct screening and awareness programs to improve worker's health. Besides, this review will highlight an urgent need for further research on hypertension in cotton textile workers that fill in some of the data gaps such as the trends and determinants of hypertension. The review findings will be made publicly available. The results of the review will be disseminated through peer-reviewed publications and presenting at professional conferences either in the form of oral or poster presentations. Any 
amendments made to this protocol when conducting the study will be outlined and reported in the final manuscript.

\section{Abbreviations}

HTN: Hypertension; DALYS: disability adjusted life years; CVD: cardiovascular disease; LMICs: LowerMiddle-Income Countries; DM: Diabetes Mellitus; PRISMA: Preferred Reporting Items for Systematic Reviews and Meta-analyses

\section{Declarations}

\section{Ethics approval and consent to participate}

Not applicable

\section{Consent for publication}

Not applicable

\section{Availability of data and materials}

Not applicable

\section{Competing interests}

The authors declare that they have no competing interests.

\section{Funding}

Not applicable

\section{Authors' contributions}

NA has conceived and designed the study. NA has drafted the manuscript and is the guarantor of the systematic review. AF \& NA has developed the search strategy and methods of the systematic review. AF \& NA has extensively review the manuscript and incorporated intellectual inputs. All authors have read, and approved the final version of the manuscript.

\section{Acknowledgements}

The authors would like to thanks Ms. Salima Farooq, Senior Instructor, School of Nursing and Midwifery Department, The Aga Khan University, Pakistan, for providing supervision as a third reviewer in case of any disagreement between two reviewers.

\section{References}


1. Forouzanfar, M.H., et al., Global burden of hypertension and systolic blood pressure of at least 110 to 115 mm Hg, 1990-2015. Jama, 2017. 317(2): p. 165-182.

2. World Health Organization. Global Health Observatory (GHO) data. Raised blood pressure, Situation and trends. https://www.who.int/gho/ncd/risk_factors/blood_pressure_prevalence_text/en/.

3. Chobanian, A.V., et al., The seventh report of the joint national committee on prevention, detection, evaluation, and treatment of high blood pressure: the JNC 7 report. Jama, 2003. 289(19): p. 25602571.

4. World Health Organization (WHO, 2019). Hypertension; Key-facts. https://www.who.int/newsroom/fact-sheets/detail/hypertension.

5. Bank W. World Bank Country and Lending Groups. LOWER-MIDDLE-INCOME ECONOMIES $(\$ 1,006$ TO \$3,955) 2018.

6. Perkovic, V., et al., The burden of blood pressure-related disease: a neglected priority for global health. Hypertension, 2007. 50(6): p. 991-997.

7. Sarki, A.M., et al., Prevalence of hypertension in low-and middle-income countries: a systematic review and meta-analysis. Medicine, 2015. 94(50).

8. Chiolero, P.B.A., Prevalence and control of hypertension. THE LANCET, 2018. 392(10155): p. 13051306.

9. Roth, G.A., et al., Global, regional, and national burden of cardiovascular diseases for 10 causes, 1990 to 2015. Journal of the American College of Cardiology, 2017. 70(1): p. 1-25.

10. Horsa, B.A., Y. Tadesse, and E. Engidawork, Assessment of hypertension control and factors associated with the control among hypertensive patients attending at Zewditu Memorial Hospital: a cross sectional study. BMC research notes, 2019. 12(1): p. 152.

11. Ofori, S.N. and J. Obosi, Prevalence of hypertension among office workers in a multi-national company in the Niger-Delta with the 2017 American College of Cardiology/American Heart Association Blood Pressure Guidelines. Preventive medicine reports, 2019. 15: p. 100899.

12. World Health Organization. A global brief on hypertension. WHO/DCO/WHD/2013.2. Geneva: WHO 2013. Available from

http://www.who.int/cardiovascular_diseases/publications/global_brief_hypertension/en/.

13. Forstater M, Implications of the global financial and economic crisis on the textile and clothing sector. International Labour Organization. International Labour Office, Geneva, 2009.

14. Bullón, J., et al., Manufacturing processes in the textile industry. Expert Systems for fabrics production. 2017.

15. Moses, J.J. and L. Ammayappan, Growth of textile industry and their issues on environment with reference to wool industry. Asian Dyer, 2006. 3: p. 61-67.

16. Nafees, A.A., et al., Pattern and predictors for respiratory illnesses and symptoms and lung function among textile workers in Karachi, Pakistan. Occup Environ Med, 2013. 70(2): p. 99-107. 
17. Ali, N.A., et al., Dose-response of Cotton Dust Exposure with Lung Function among Textile Workers: MultiTex Study in Karachi, Pakistan. The international journal of occupational and environmental medicine, 2018. 9(3): p. 120.

18. ZIA, S., S. JAVEED, and F. JAN, Pathophysiological Effects of Cotton Dust Pollution on Blood Pressure in Textile Workers. Cell. 300: p. 6617901.

19. Suryakar, A., et al., A study of oxidative stress in cotton industry workers from Solapur city. Biomedical Research, 2010. 21(3): p. 260-264.

20. Rodrigo, R., et al., Relationship between oxidative stress and essential hypertension. Hypertension Research, 2007. 30(12): p. 1159.

21. Briones, A.M. and R.M. Touyz, Oxidative stress and hypertension: current concepts. Current hypertension reports, 2010. 12(2): p. 135-142.

22. Ismail, I.M. and K. Binub, Prevalence of hypertension and its associated factors among cotton textile workers of Kannur, Kerala. Menoufia Medical Journal, 2016. 29(4): p. 991.

23. Bhowmik, B., et al., Obesity and associated type 2 diabetes and hypertension in factory workers of Bangladesh. BMC research notes, 2015. 8(1): p. 460.

24. Rizi, H.A.Y. and H. Dehghan, Effects of occupational noise exposure on changes in blood pressure of workers. ARYA Atherosclerosis, 2013: p. 183-186.

25. Starzyński, Z., et al., Incidence of arterial hypertension in the population of workers in the textile industry in Lódź. Medycyna pracy, 1985. 36(2): p. 131-138.

26. Tiwai, R., et al., Hypertension among cotton textile workers. Indian journal of public health, 2003. 47(1): p. 34-36.

27. Sumardiyono, S., et al., The Association between Risk Factors and Blood Pressure in the Textile Industry Workers. Global Medical \& Health Communication, 2017. 5(3): p. 228-235.

28. Kitronza, P.L. and P. Mairiaux, Occupational stress among textile workers in the Democratic Republic of Congo. Tropical medicine and health, 2015.

29. Koskela, R., M. Klockars, and E. Järvinen, Mortality and disability among cotton mill workers. Occupational and Environmental Medicine, 1990. 47(6): p. 384-391.

30. Luckhaupt, S.E., et al., Prevalence of obesity among US workers and associations with occupational factors. American journal of preventive medicine, 2014. 46(3): p. 237-248.

31. Safety, A. and C. Council, Overweight and Obesity: Implications for Workplace Health and Safety and Workers' Compensation. 2008: Australian Safety and Compensation Council.

32. Di Lorenzo, L., et al., Effect of shift work on body mass index: results of a study performed in 319 glucose-tolerant men working in a Southern Italian industry. International journal of obesity, 2003. 27(11): p. 1353.

33. Bloom, D.E., et al., From burden to" best buys": reducing the economic impact of non-communicable disease in low-and middle-income countries. 2011, Program on the Global Demography of Aging. 
34. Moher, D., et al., Preferred reporting items for systematic review and meta-analysis protocols (PRISMA-P) 2015 statement. Systematic reviews, 2015. 4(1): p. 1.

35. Xiehang, C.D.L., Evaluation and prospect of reference management software--a case study of EndNote and NoteExpress. New Technology of Library and Information Service, 2009: p. Z1.

36. Sterne, J.A., et al., ROBINS-I: a tool for assessing risk of bias in non-randomised studies of interventions. bmj, 2016. 355: p. i4919.

\section{Tables}

Table 1 Eligibility criteria 


\begin{tabular}{|c|c|c|}
\hline sute & Inclusion Criteria & Exclusion Criteria \\
\hline ation & $\begin{array}{l}\text { Studies involving adult cotton textile } \\
\text { workers of both genders }\end{array}$ & $\begin{array}{l}\text { Studies involving target groups of adult workers working in } \\
\text { another factory mill (oil mill, steel mill and coal mill) }\end{array}$ \\
\hline of & $\begin{array}{l}\text { Observational studies (survey, } \\
\text { descriptive, cross-sectional cohort and } \\
\text { case control study and brief } \\
\text { communication studies having full- } \\
\text { text article) }\end{array}$ & $\begin{array}{l}\text { RCT and quasi-experimental study, Qualitative formative } \\
\text { studies, systematic reviews case studies, protocols, } \\
\text { commentaries, editorials, unpublished articles, symposium } \\
\text { proceedings, conference abstracts and irretrievable } \\
\text { documents }\end{array}$ \\
\hline me & $\begin{array}{l}\text { Studies demonstrating outcomes } \\
\text { related to prevalence, incidence along } \\
\text { with risk factor of hypertension }\end{array}$ & $\begin{array}{l}\text { Studies demonstrating outcomes in workers having } \\
\text { different comorbidity }\end{array}$ \\
\hline $\lg$ & Studies conducted in LMICs & Studies conducted elsewhere \\
\hline lage & $\begin{array}{l}\text { Studies available in the English } \\
\text { Language }\end{array}$ & Studies in other languages \\
\hline $\mathrm{d}$ & $\begin{array}{l}\text { Studies published between the period } \\
\text { of } 1 \text { January, } 2000 \text { to } 31 \text { December, } \\
2019\end{array}$ & Studies published out of the period of 2000 to 2019 \\
\hline Irces & $\begin{array}{l}\text { Systematic literature search on the } \\
\text { major electronic databases including } \\
\text { PubMed, CINAHL Plus, Science } \\
\text { Direct, and Cochrane }\end{array}$ & $\begin{array}{l}\text { Systematic literature search other than these electronic } \\
\text { databases including PubMed, CINAHL Plus, Science Direct, } \\
\text { and Cochrane }\end{array}$ \\
\hline
\end{tabular}

Table 2: Search strategy 


\begin{tabular}{|l|l|}
\hline Population & $\begin{array}{l}\text { ('textile* [Mesh] OR 'workers*' OR 'factory worker*' OR 'mill worker'* OR 'male worker*'OR 'cotton } \\
\text { worker*' [Mesh]) AND }\end{array}$ \\
\hline Exposure & $\begin{array}{l}\text { ('Risk-factors' OR 'factors' OR 'predictors' OR 'prevalence' OR 'incidence' 'risks', 'prevalence and } \\
\text { factors') AND }\end{array}$ \\
\hline Outcome & Outcome ( 'hypertension' OR 'blood pressure' OR 'systolic blood pressure' OR 'diastolic blood \\
\hline Setting & Studies conducted in LMIC \\
\hline Filters & Publication date from 1 January, 2000 to 31 December, 2019; Humans \\
\hline
\end{tabular}

Table 3 Data Extraction Table

\begin{tabular}{|c|c|c|c|c|c|c|c|c|}
\hline racteristics & $\begin{array}{l}\text { Study } \\
1\end{array}$ & $\begin{array}{l}\text { Study } \\
2\end{array}$ & $\begin{array}{l}\text { Study } \\
3\end{array}$ & Study 4 & Study 5 & Study 6 & Study 7 & Study 8 \\
\hline \multicolumn{9}{|c|}{ dy Author \& Year } \\
\hline \multicolumn{9}{|c|}{ ting \& Country } \\
\hline \multicolumn{9}{|l|}{ dy Aim } \\
\hline \multicolumn{9}{|l|}{ dy Design } \\
\hline \multicolumn{9}{|c|}{ dy Population } \\
\hline \multicolumn{9}{|l|}{ le proportion } \\
\hline \multicolumn{9}{|l|}{ rage age } \\
\hline \multicolumn{9}{|l|}{ rage $\mathrm{BP}$} \\
\hline \multicolumn{9}{|c|}{ Measurement methods } \\
\hline \multicolumn{9}{|c|}{$\begin{array}{l}\text { Measurement } \\
\text { Iaratus }\end{array}$} \\
\hline \multicolumn{9}{|l|}{ valence } \\
\hline \multicolumn{9}{|l|}{ k-Factors } \\
\hline Morbidities & & & & & & & & \\
\hline
\end{tabular}


Studies identified through database searching

$(n=)$

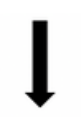

Studies included by screening relevant titles after de-duplication

$$
(n=)
$$

Studies included by screening abstract $(n=)$
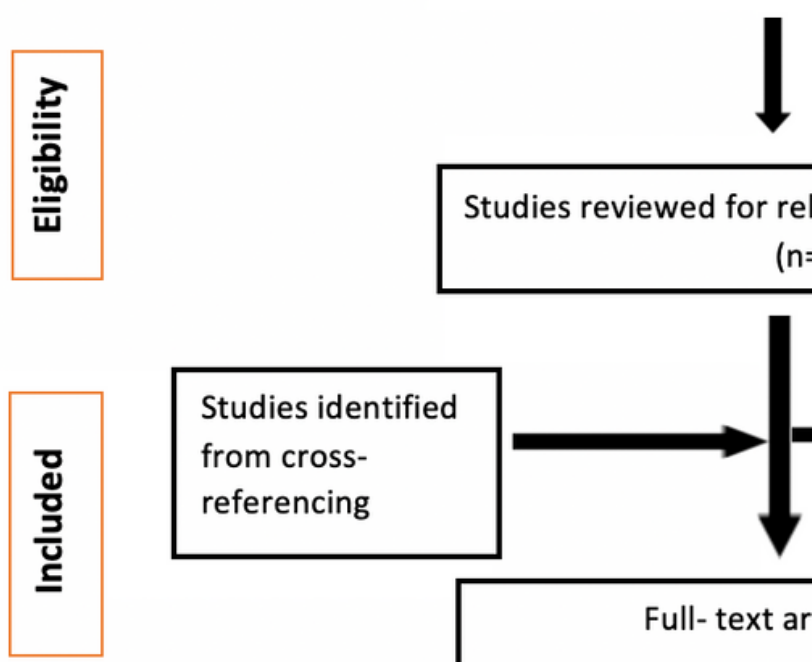

Studies reviewed for relevant full-text papers $(n=)$

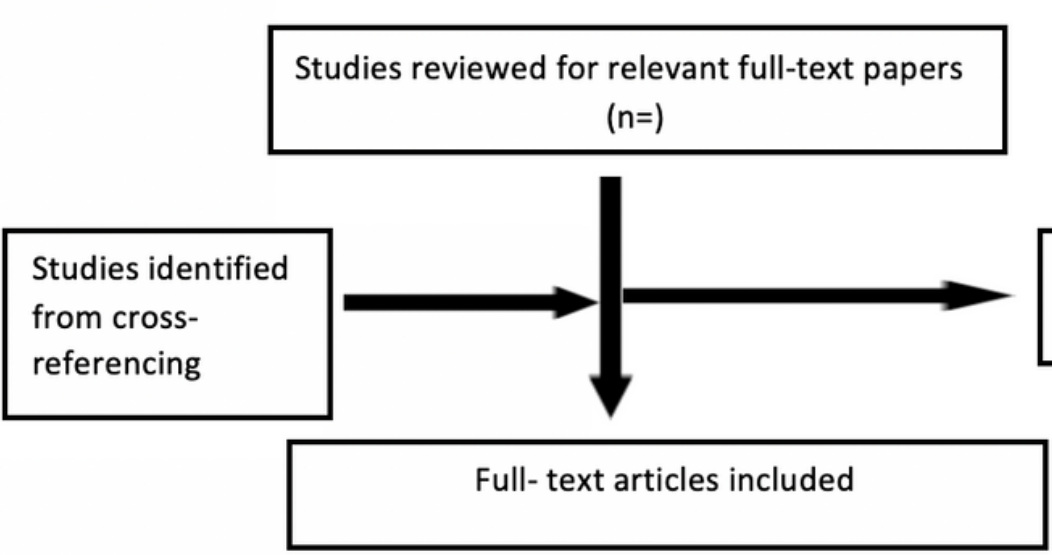

Duplication excluded

$(n=)$

Titles excluded for not meeting the eligibility criteria

$(n=)$

Abstracts excluded for not meeting the eligibility criteria

( $n=)$

Abstracts unavailable

( $n=)$

\section{Figure 1}

PRISMA Flow Diagram for Database Search of Studies

\section{Supplementary Files}

This is a list of supplementary files associated with this preprint. Click to download.

- Additionalfile3DataExtractionTable.docx

- Additionalfile1PRISMAPchecklist.doc

- Additionalfile2SearchStrategy.docx

Full text excluded for not meeting the eligibility criteria $(n=)$ 